\title{
Impact of Food Simulants Material on Orthodontic Bond Strength after Application of Caries Infiltrant Resin (In Vitro Study)
}

\author{
Ahmed Muhsin Yousif AL-Mayali ${ }^{1}$, Mohammed Nahidh ${ }^{2}$, Hayder A. Kadhim ${ }^{3}$, Ali Hadi Fahad ${ }^{4}$ \\ ${ }^{1}$ Lecturer, Pediatric Dentistry, Orthodontics \& Prevention dentistry department, Dentistry College, Kufa University \\ /Iraq, ${ }^{2}$ Assist. Prof., Orthodontic department, Dentistry College, University of Baghdad/Iraq, ${ }^{3}$ Lecturer, Pediatric \\ dentistry, Orthodontics \& Prevention dentistry department, Dentistry College, Kufa University /Iraq, ${ }^{4}$ Assist. Prof., \\ Pediatric dentistry, Orthodontics \& Prevention dentistry department, Dentistry College, Kufa University/Iraq
}

\begin{abstract}
Objective: To investigate the effect of using different food simulant materials on the shear bond strength (SBS) of orthodontic bracket after Icon application. DESIGHN: Laboratory study. SETTING: Pediatric dentistry, Orthodontics \& Prevention dentistry department, Dentistry College, Kufa University \& department of materials, Technology university.

Methods: Metal brackets were bonded to 48 extracted premolars and were randomly arrange for three categories with 16 teeth for each, revealing the storage solutions, which were distilled water, 50\% aqueous ethanol (alcoholic food), and corn oil (fatty food). Then each category was split for two subgroups, with 8 teeth describing Icon application, all specimens are immersed in their corresponding food simulants for 30 days. After the completed proposed time, the specimens were debonded by using an Instron apparatus to test the SBS. Results: Results showed significantly diminished the bond strength of orthodontic adhesive after holding the specimens for 30 days in the food simulants specially ethanol (highest for $50 \%$ ethanol, less for corn oil \& least for D.W). Icon categories showed higher SBS rather than non-Icon categories. Conclusion: Application of Icon on enamel will increase the bond strength $\&$ the food simulating agents especially food and rinses containing ethanol could influence the orthodontic bond strength which causes bond failure so that the clinicians should inform their patients concerning their possible adverse effects and complications.
\end{abstract}

Key Words: Orthodontics, Food simulants, bonding strength, Resin Infiltration; Icon, caries infiltrant resin, White spot lesion.

\section{Introduction}

Orthodontic treatment is mostly accomplishing with fixed orthodontic appliances \& establish significantly higher WSL than normal persons, because oral hygiene is more ambitious in patients with numerous-bracket orthodontics than in patient without fixed appliance ${ }^{(1)}$. Pervasiveness of WSLs is relatively huge, affecting more than $25 \%$ of the patients earning orthodontic treatment,

\section{Corresponding author:}

Ahmed Muhsin Yousif AL-Mayali

ahmedalmayali@yahoo.co.uk capturing at least one new lesion over the treatment ${ }^{(2)}$. Demineralization may occur rapidly, as fast as in period 4 weeks after the placement of brackets ${ }^{(3)}$.

More recently, a minimum invasive treatment pathway was introduced, in which the WSL is infiltrated adopting a low-viscosity resin. When tooth surface been carried out by the infiltration resin with demand for an adhesive method like: using orthodontic brackets \& when the used infiltration material that not be detached, the question: Is there bonding force between the treated exterior of the tooth and the unity of this material to the used bonding scheme? ${ }^{(4)}$. The aim of this study was to study the effect of using different food simulant materials 
on the SBS of orthodontic bracket after utilization of Icon.

\section{Marerial \& Methods}

In this our study, we used seventy extracted intact upper first premolars ${ }^{(5)}$. Forty-eight teeth were elected after being inspected by magnifying lens ${ }^{(6)}$. The elected teeth must be:

\section{1-Caries free.}

2-Free of enamel cracks or restorations.

3-Without developmental anomalies.

Standard edgewise S.S. orthodontic bracket (Ortho Technology, USA) and a light cure composite adhesive (3M, USA) were used in this study.

The food simulants were those approved by the Food and Drug Administration in U.S.A. ${ }^{(7)}$ :

1. Beverages including alcoholic drinks simulated by $50 \%$ aqueous ethanol solutions.

2. Fatty food including butter, fatty meats and vegetable oils simulated by corn oil.

3. Water established using distilled water.

The possessed teeth were split into three categories, containing sixteen teeth each, according to the type of storage solution, which are distilled water, $50 \%$ aqueous ethanol (alcoholic food), and corn oil (fatty food). Then each category was split for two subgroups with eight teeth for each, according to Icon application.

A two metal plates with L-shape specially made for this purpose, were painted with a thin layer of separating medium (Vaseline) and placed opposite to each other forming a box around the vertically positioned teeth. Then the acrylic (Vertex, Netherlands) was poured till reach the cemento-enamel junction of the teeth ${ }^{(8)}$. After mounting, the buccal exterior was scrub by a rubber cup $\&$ pumice which is free from fluoride for $10 \mathrm{~s}{ }^{(9)}$. The teeth were flush by water for $10 \mathrm{~s}$. and then dry with air. This strategy was set to mimic the 'real life' of clinical condition ${ }^{(10)}$. Etching by using Phosphoric acid (37\%) on the exterior of the crown as long as $30 \mathrm{~s}$. after that rinsed with water, then dried with air till the exterior crown marked as chalky ${ }^{(11)}$.

\section{BONDING PRECEDURE WITHOUT ICON APPLICATION:}

The 7 th generation bond (3M company, USA) was used then orthodontic adhesive was administered to the S.S. brackets ${ }^{(12)}$. Which was then fixed in the midst third of the buccal exterior, the extra composite was removed by using dental probe ${ }^{(13)}$. The adhesive composite was curing up to $40 \mathrm{~s}{ }^{(14)}$ utilizing LED curing device with a range $450-550 \mathrm{~nm}$.

\section{BONDING PRECEDURE WITH ICON APPLICATION:}

This done by using (Icon-Dry, DMG, Hamburg, Germany). Apply Etch for 2 min. next washed by water, after that dry with air, Handle Icon-dry $30 \mathrm{sec}$. and check it by visual inspection, then dry air. Handle IconInfiltrant, to $3 \mathrm{~min}$. give away by air then curing for 40 sec. Infiltrant was applied for $1 \mathrm{~min}$. clear away extra infiltrant then curing for $40 \mathrm{sec}$. The 7 th generation bond (3M, USA) was used then orthodontic adhesive (3M, USA) was applied.

Then, all tested categories were sinking in distilled water or to the food simulant according to their category, and preserve it in the incubator at $37^{\circ} \mathrm{C}$ for 30 days ${ }^{(15)}$.

After 30 days, they ready for the SBS that was done by an Instron universal machine (Laryee, $50 \mathrm{KN}$, China), a defined speed was $0.5 \mathrm{~mm} / \mathrm{min}$. ${ }^{(16)}$. The loading fail (Newton) / paltry of bracket $\left(10.90 \mathrm{~mm}^{2}\right)$ to obtain the SBS in MPa. Statistical analysis was implement with applying SPSS software Version 21.

\section{Results}

The SBS (MPa) descriptive statistics and the effectiveness of food simulants material \& Icon usage on the SBS were calculated (Table 1).

Anova test was performed to examine the difference between each three types of storage medium; SBS reduction was high (highly significant) for corn oil group $\&$ highest (highly significant) for 50\% ethanol alcohol group and was the least reduction for D.W. group (Table 2).

Comparison of SBS of adhesive \& Descriptive statistics according to the Icon application was calculated. Mean rate of SBS were highest (highly significant) in 
Icon category in comparison with the other category without Icon application as displayed in (Table 3).

\section{Discussion}

This study was performed to investigate the response to aqueous foods, alcoholic foods, and fatty foods as represented by dietary simulating liquids (D.W, corn oil \& 50\% aqueous ethanol) and their effect upon the SBS of orthodontic adhesive bonding material with the presence of caries infiltrant resin (Icon).

The treatment of WSL by using infiltrating instead of or postponing conventional restorative treatment may have a positive impact on good oral health as it helps to increase in life cycle of a tooth ${ }^{(17)}$.

Orthodontic treatment based on a sufficient bond strength of brackets to enamel. To withstand normal orthodontic force, the minimal bonding strength should be between 6 and $8 \mathrm{MPa}{ }^{(18)}$.

This is in vitro methods that simulating oral condition have been designed to mimic the clinical performance. Because the difficulty in vivo of oral environments can be partially be simulated in vitro, so there is no in vitro test can be to mimic the complex processes found in vivo ${ }^{(19)}$. in vivo evaluation, it is very complicated, time consuming, expensive $\&$ the most important issue is the ethical reasons. Thus, Continuous exposure of solution was preferred to hasten the resistant effect of the evaluated material ${ }^{(20)}$. This may prompt solution to be invasive more into the specimens. Intermittent exposure to a resistant agent will causes low invasion rate because simulant desorption while disclosure. The material may not fully desorb due to the force established by the invasion gradient to continue invasion into the specimens ${ }^{(21)}$. in clinical conditions the agent may either be absorbed by the adherent detritus at the interfacial margins or generated by decomposition (22). The distilled water group show the high SBS because it is not very susceptible by chemical breakdown. Lee et al show that when exposure to artificial saliva (90-95\% water) for 30 days of engagement will not effect on bond strength ${ }^{(23)}$.

A high significant result in SBS of the specimens immersed in food simulants with 50\% aqueous ethanol showed the less in bond strength. This is in agreement with several previous studies ${ }^{(15)}$.

The ethanol has solvent properties as cross linked dimethacrylate resins are virtually insoluble, in spite of that its capable of swelling in good solvents ${ }^{(24)}$. The ethanol which dispersed into the composite may result in micro-crack in architecture that causes bonding weakness. Ethanol has dissolving feature similar to Bis-GMA and this may farther improve its seepage into the composite leading to farther destruction ${ }^{(25)}$. The diffusion properties of the ethanol solution in dental composite specimens is much more than that of water (26).

Clinically, intra-oral exposure to ethanol either from alcoholic mouth washes or from drink. Usually, orthodontists prescribe mouth washes for their patients for the whole treatment time to keep high oral hygiene (27).

Apply of peppermint oil to assist in orthodontic bond and resin elimination ${ }^{28)}$. The significant maceration of resin can occur after continuous exposure to the oil ${ }^{(29)}$. The time duration being clinically offensive. The 1-hour liability to the oil may cause decrease in bond strength, However, the oil remarkably loud up of ARI scores, display fewer resin on the enamel exterior, which could helpful in 'clean up' next to bond elimination ${ }^{(15)}$.

According to our result the Icon group show high SBS (high significant). By using infiltration to help for protection against demineralization, the using of resin infiltration with multi adhesive systems used in bonding seems to be feasible, this let us go ahead with fixed orthodontic treatment after using infiltration. moreover, there were seems to be strengthen performance of adhesives bond ${ }^{(30,31)}$.

The Icon is a useful to enhance the SBS of brackets to the enamel exterior ${ }^{27)}$. The effect of Icon on the SBS of resin cements that used on sound and demineralized enamel. The Icon elevated the SBS of most resin cements types while decline the possibility of enamel fracture during debonding ${ }^{32}$. The pair of above studies are compatible with our SBS integrity of Icon.

The Icon have a hydrophilic trait that empower their seepage into the tooth exterior which results in a direct connection to this tooth exterior. This will diminish the 
effectiveness of $\mathrm{O} 2$ and enhance the polymerization, in turn empower the SBS ${ }^{(33)}$. Even on intact enamel, the using of Icon had supplementary action on SBS, possibly as an action of the excessive moisten properties of the TEGDMA resin ${ }^{(34)}$. It's clear that the Icon may be used on the tooth exterior which not skeptically hamper the bond betwixt resin composite and enamel exterior. It displayed to be statistically preferable rather than other categories .

\begin{tabular}{|c|c|c|c|c|c|c|c|}
\hline \multirow{2}{*}{ Bonding Types } & \multirow{2}{*}{ Food Simulants } & \multirow{2}{*}{ No. } & \multicolumn{2}{|c|}{ Shear Bond } & \multicolumn{3}{|c|}{ Statistical Differences } \\
\hline & & & Mean & SD & F- value & $\mathrm{df}$ & p-value \\
\hline \multirow{3}{*}{ ICON } & Alcohol & 8 & 9.14 & 0.971 & \multirow{3}{*}{19.118} & \multirow{3}{*}{2} & \multirow{3}{*}{$>0.001 * *$} \\
\hline & Corn Oil & 8 & 11.23 & 0.835 & & & \\
\hline & D.W & 8 & 13.66 & 2.191 & & & \\
\hline \multirow{3}{*}{ Without ICON } & Alcohol & 8 & 6.70 & 1.133 & \multirow{3}{*}{21.267} & \multirow{3}{*}{2} & \multirow{3}{*}{$>0.001 * *$} \\
\hline & Corn Oil & 8 & 8.43 & 0.915 & & & \\
\hline & D.W & 8 & 10.06 & 1.035 & & & \\
\hline
\end{tabular}

Table 1. Mean and S.D values of SBS among food stimulants in relation to bonding type with statistical differences.

Table 2. Statistical differences of shear bond strength among food stimulants in relation to bonding types.

\begin{tabular}{|c|c|c|c|c|c|}
\hline \multirow{2}{*}{ Food Simulants } & \multirow{2}{*}{ Bonding Types } & \multirow{2}{*}{ No. } & \multicolumn{3}{|c|}{ Shear Bond Strength } \\
\hline & & & t-value & df & p-value \\
\hline \multirow{2}{*}{ Alcohol } & $\mathrm{ICON}$ & 8 & \multirow{2}{*}{4.621} & \multirow{2}{*}{14} & \multirow{2}{*}{$>0.001^{* *}$} \\
\hline & Without ICON & 8 & & & \\
\hline \multirow{2}{*}{ Corn Oil } & ICON & 8 & \multirow{2}{*}{6.397} & \multirow{2}{*}{14} & \multirow{2}{*}{$>0.001^{* *}$} \\
\hline & Without ICON & 8 & & & \\
\hline \multirow{2}{*}{ D.W } & ICON & 8 & \multirow{2}{*}{4.202} & \multirow{2}{*}{14} & \multirow{2}{*}{$0.001^{* *}$} \\
\hline & Without ICON & 8 & & & \\
\hline
\end{tabular}


Table 3. Mean and standard deviation values of bonding types with statistical difference.

\begin{tabular}{|l|l|l|l|l|l|l|}
\hline & \multicolumn{7}{l}{ No. } & \multicolumn{2}{l}{ Shear Bond Strength } & \multicolumn{2}{l|}{ Shear Bond Strength } \\
& Bonding Types & Mean & SD & t-value & df & p-value \\
\hline ICON & 24 & 11.34 & 2.351 & & \multirow{2}{*}{$0.001^{* *}>$} \\
\hline Without ICON & 24 & 8.40 & 1.714 & & 46 & \\
\hline
\end{tabular}

\section{Conclusion}

1-According to our result, the storage in food simulants for 30 days ( $50 \%$ aqueous ethanol \&corn oil) produced a significant reduction in SBS.

1-The Icon application can be used on enamel exteriors even before bonding orthodontic brackets as improve bonding $\&$ used to inhibit demineralization.

3-The use of alcohol containing mouth washes may cause premature debonding of brackets especially at the beginning of treatment. So it can be rational to instruct the patients to fend off alcoholic mouth rinses.

4- The use of alcohol containing mouth washes had advantage by making the debonding easier at the end of orthodontic treatment.

Ethical Clearance: The Research Ethical Committee at scientific research by ethical approval of both $\mathrm{MOH}$ and MOHSER in Iraq

Conflict of Interest: Non

Funding: Self-funding

\section{References}

1. Bishara, S.E. and A.W. Ostby. White Spot Lesions: Formation, Prevention, and Treatment. Semin orthod.2008; 14(3): 174-182.

2. Lucchese A, Gherlone E. Prevalence of white-spot lesions before and during orthodontic treatment with fixed appliances. European Journal of Orthodontics.2013; 35:664-668.

3. Bergstrand F, Twetman S. A review on prevention and treatment of post-orthodontic white spot lesions - evidence-based methods and emerging technologies. The open dentistry journal.2011; 5:158-162.

4. Gauthier, M. A., et al. Oxygen inhibition in dental resins. Journal of dental research.2005; 84: 725-29.

5. Vicente, Ascensión, and Luis Alberto Bravo. Shear bond strength of precoated and uncoated brackets using a self-etching primer: An in vitro study. Angle orthodontist.2007; 77: 524-27.

6. D'Attilio, Michele, et al. Shear bond strength, bond failure, and scanning electron microscopy analysis of a new flowable composite for orthodontic use. Angle orthodontist. 2005;75: 410-15.

7. United States Food and Drug Administration Guideline for Chemistry and Technology. Requirement of indirect additive petition. US Food and Drug Administration,1988.

8. Rajagopal, Rangaswamy, Sridevi Padmanabhan, and Janakirama Gnanamani. A comparison of shear bond strength and debonding characteristics of conventional, moisture-insensitive, and selfetching primers in vitro. Angle orthod.2004; 74: 264-68.

9. Bishara, Samir E., et al. A new premixed self-etch adhesive for bonding orthodontic brackets. Angle orthodontist.2008; 78: 1101-04.

10. Gronberg, Kimberly, et al. Distance and time effect on shear bond strength of brackets cured with a secondgeneration light-emitting diode unit. Angle orthodontist.2006; 76: 682-88.

11. Katona, Thomas R., and Robert W. Long. Effect of loading mode on bond strength of orthodontic brackets bonded with 2 systems. American journal of orthodontics and dentofacial orthopedics.2006; 129: 60-64.

12. Vorhies, A. Bronwen, et al. Enamel demineralization adjacent to orthodontic brackets bonded with 
hybrid glassionomer cements: An in vitro study. American journal of orthodontics and dentofacial orthopedics.1998;114: 668-74.

13. McLaughlin, Richard P., John C. Bennett, and Hugo J. Trevisi. Systemized Orthodontic Treatment Mechanics. Elsevier health sciences.2001; 68.

14. Ewoldsen, Nels, et al. Effects of enamel conditioning on bond strength with a restorative lightcured glass ionomer. Journal of clinical orthodontics.2005; 29: 621-24.

15. Hobson RS, McCabe JF, Hogg SD. The effect of food simulants on enamel-composite bond strength. Br j orthod .2000; 27(1): 55-9.

16. Sharma-Sayal SK, Rossouw PE, Kufkami GV, Titley KC. The influence of orthodontic bracket base design on shear bond strength. Am j orthod dentofacial orthop.2003;124(1): 74-82.

17. Schwendicke, F., et al. Costs and effectiveness of treatment alternatives for proximal caries lesions. PLoS one.2014; 9 (1): e86992.

18. Reynolds J R. A review of direct orthodontic bonding. British journal of orthodontics.1975;2:171 -178 .

19. Oilo G. Biodegradation of dental composites/glassionomer cements' Adv dent res.1992;6: 50-4.

20. Lee SY, Greener EH, Covey DA, Menis DL. Effect of food/oral simulating fluids on microstructure and strength of dentine bonding agents. $\mathrm{J}$ oral rehabil.1996;23(5): 353-61.

21. Lee SY, Lin CT. Storage effect on dentine structure and on resultant composite bond strengths. J oral rehabil .1997;24(11): 823-34.

22. Lee SY, Huang HM, Lin CY, Shih YH. Leached components from dental composites in oral simulating fluids and the resultant composite strengths.J oral rehabil .1998; 25(8): 575-88.

23. Akram F. Alhuwaizi \& H.Yousif. The effect of food simulants on the bond strength of orthodontic adhesive. Iraqi orthod journal.2008; 4(1): 1-7.

24. McKinney JE, Wu W. Chemical softening and wear of dental composites. J dent res.1985; 64(11):132631.
25. Lee SY, Greener EH, Mueller HJ, Chiu CH. Effect of food and oral simulating fluids on dentine bond and composite strength. J dentistry.1994;22(6): 352-9.

26. Kalachandra S. Influence of fillers on the water sorption of composites. Dent mater .1989;5(4): 283-8.

27. Attin R, Stawarczyk B, Kecik D, et al. Shear bond strength of brackets to demineralize enamel after different pretreatment methods. Angle orthod.2012;82:56-61.

28. Waldron, M. and Causton, B. E. A study of the fracture toughness of a light cured composite. Journal of dental research.1991; 70: 696.

29. Larmour, C. J. and Chadwick, R. G. Effects of a commercial orthodontic debonding agent upon the surface micro-hardness of two orthodontic bonding resins. Journal of dentistry.1995; 23: 37-40.

30. Ahmed M. Yousif Al-Mayali. Effect of Icon and bond type on shear bond strength (An in vitro study). International journal of medical research \& health sciences.2017; 6(11): 58-66.

31. Wisam W. Alhamadi, Essam Osman, Fayez Saleh, Ahmed M. AL-Mayali. Effect of Resin Infiltrant Pretreatment on Shear Bond Strength of Metal Orthodontic Brackets in Vitro Study. International journal of dentistry and oral health.2018; 4 :105110.

32. Naidu E, Stawarczyk B, Tawakoli PN, et al. Shear bond strength of orthodontic resins after caries infiltrant preconditioning. Angle orthod .2013; 83: 306-312.

33. Sano, Hidehiko, et al. Relationship between surface area for adhesion and tensile bond strength evaluation of a micro-tensile bond test. Dental materials. 1997; 10: 236-40.

34. Paris, Sebastian, et al. Penetration coefficients of commercially available and experimental composites intended to infiltrate enamel carious lesions. Dental materials.2007;23: 742-48. 